Dr. Miguel Ángel Martín Valmayor

Universidad Francisco de Vitoria

@ miguel.martin@ufv.es
(iD) $0000-0003-4522-9960$
Recibido / Received 17 de noviembre de 2018

- Aceptado / Acepted 28 de enero de 2019

- Páginas / Pages De la 129 a la 138

- ISSN: 2531-0054

\title{
Diseño de un modelo de análisis de comportamiento estratégico en la economía digital
}

\begin{abstract}
Design of a model of analysis of strategic behavior in the digital economy
El propósito de este estudio es avanzar en el análisis de las decisiones empresariales y en la valoración de su comportamiento, comparando la coherencia de los resultados generados a posteriori frente a las estrategias planteadas a priori. Para ello, se aplicará una técnica de análisis multicriterio de predicción de comportamiento del ámbito agrario a un entorno empresarial, modelizada matemáticamente bajo un esquema de sostenibilidad a largo plazo, con cinco objetivos corporativos diferentes (crecimiento, solvencia, creación de valor, dividendo y eficiencia operativa). Este planteamiento se ejemplificará con un caso de estudio de una empresa del sector digital y se valorarán los resultados obtenidos por esta y el modelo seguido a lo largo de los últimos años.
\end{abstract}

PALABRAS CLAVE: planificación estratégica, finanzas, optimización multicriterio, análisis de decisiones, análisis de comportamiento.

The purpose of this study is to advance in the analysis of business decisions and the assessment of their behavior, comparing the coherence of the «a posteriori» results obtained from the «a priori» strategies proposed. For this, a multicriteria analysis technique for predicting the behavior in the agriculture industry will be extended to this business environment. This problem will be mathematically modeled under a long-term sustainability structure with five different corporate objectives (growth, solvency, value creation, dividend and operational efficiency). This approach will be exemplified with a case study of a leading company in the digital sector and the results obtained over the last few years will be evaluated.

KEYWORDS: strategic planning, finance, multi-criteria decision making, decision analysis, behavioral analysis.

\section{Introducción}

En el ámbito de la teoría de la decisión, las técnicas multicriterio han alcanzado hoy día un alto nivel de desarrollo y se aplican de forma general en multitud de medios. Permiten 
formalizar y resolver racionalmente problemas de toma de decisiones optimizando de forma conjunta criterios múltiples en conflicto.

El germen inicial de las teorías multicriterio se encuentra en el desarrollo de Koopmans [1], en el que aparece el concepto matemático de «vector eficiente», que, junto con el de Kuhn-Tucker [2], donde se deducen las condiciones que garantizan la existencia de soluciones eficientes para problemas de programación vectorial, permitieron a Charnes, Cooper y Ferguson definir el concepto de «programación por metas» [3] como la estrategia que permite la búsqueda de soluciones satisfactorias capaces de minimizar de forma conjunta el error de aproximación. Posteriormente, el profesor Ron Howard aplicó técnicas estadísticas de forma sistemática para resolver problemas de decisiones, y acuñó el término «análisis de decisiones» en 1964.

Estas y otras aportaciones culminaron en la década de los 70 del siglo xx con la Primera Conferencia Internacional de Carolina del Sur, que se celebró en 1972 y significó el nacimiento formal de las metodologías multicriterio y permitió el desarrollo de los principales métodos de optimización multicriterio. En 1973 surge la «programación compromiso», desarrollada por los profesores Yu [4] y Zeleny [5], que formalizaron matemáticamente la búsqueda de soluciones óptimas o aquellas de menor distancia a la solución ideal que optimiza cada criterio de manera individual. Muchos de los problemas computacionales planteados se resuelven con la reciente aportación de la "programación compromiso extendida» realizada por André y Romero [6] que, bajo condiciones de continuidad y convexidad en la formulación de los objetivos, permiten linealizar soluciones de problemas con métricas complejas por medio de combinaciones lineales.

Uno de estos problemas en el ámbito de la gestión empresarial es la decisión acerca de la adecuada estructura de financiación y de su potencial impacto en el posterior desarrollo del plan de negocio. Muchos autores han construido modelos de análisis, estudiando criterios de forma individual y priorizando secuencialmente estas decisiones de manera iterativa. El planteamiento de este artículo es valorar si existe la posibilidad de evaluar el comportamiento empresarial a posteriori a partir de estos modelos. En este sentido, se pretende aplicar una metodología desarrollada por Sumpsi et al. [7] en el ámbito agrario midiendo el porcentaje de éxito alcanzado para cada objetivo a partir de los resultados observados.

\section{Revisión de la literatura}

De manera similar a las técnicas multicriterio, es a finales de los 70 y en los años 80 del siglo $x x$ cuando, gracias al desarrollo general de las herramientas computacionales, se pudieron proponer modelos financieros más complejos que permitieron eliminar los errores de cálculo aritmético. El primer modelo multicriterio de gestión aplicada surgió en 1979 con el planteamiento de Kvanli [8], que integró un modelo de planificación financiera con un esquema multicriterio de programación por metas bajo una estructura flexible de decisiones en árbol. En 1989, el trabajo recopilatorio de Batson [9] ya describía hasta 10 aplicaciones exitosas en ámbitos económico-financieros aplicando la metodología de la programación por metas. Cuestionaba como limitaciones el largo tiempo de recogida de datos y la indivisibilidad de los proyectos, que limitaban los diferentes modelos a una simple herramienta de «resolución de conflictos».

Estos desarrollos permitieron extender estos planteamientos a entornos más especializados resolviendo problemas de difícil respuesta. Así, en 1995, surge el modelo de Goedhart y 
Spronk [10] de planificación financiera con objetivos fraccionales que permitió utilizar algoritmos lineales de programación por metas a objetivos financieros. En 1997, el modelo de Maranas et al. [11] planteó el problema de distribuir activos financieros en función de categorías de inversión con funciones continuas y convexas que garantizaba la existencia de soluciones óptimas. En 1999, Tarrazo y Gutiérrez [12] modelizaron la incertidumbre futura en el ámbito de la planificación financiera empleando redes neuronales y lógica borrosa. Recientemente, Martin et al. [13] y [14] propusieron modelos de planificación estratégica de largo plazo buscando la optimización conjunta de diferentes criterios a la hora de plantear los estados financieros. En la actualidad, los desarrollos multicriterio aplicados a problemas de ámbito financiero se están centrando mayoritariamente en realizar investigación aplicada para resolver problemas concretos.

Dentro de los principales estudios bibliográficos que combinan desarrollos de decisión multicriterio en ámbitos financieros, se aprecia un ámbito de interés creciente. Zoupounidis et al. [15] actualizaron en 2015 un estudio previo de 1999, indicando más de 450 artículos de investigación en el ámbito de la gestión financiera durante el periodo 2004-2015, de los cuáles más de 300 correspondían a los últimos cinco años.

Dada la potencialidad de las técnicas de decisión multicriterio en este ámbito de la planificación financiera, cabría hacerse ahora la pregunta inversa como hipótesis de estudio. ¿Podría ser verificable que, una vez observados los resultados ofrecidos por una empresa, se pudiese valorar su estrategia a partir de estos? ¿Resultaría razonable asumir que las empresas siguiesen criterios alternativos a los de maximización del beneficio como únicos objetivos directores de su estrategia, como sostienen las teorías económicas del comportamiento?

Tomando como base de partida diferentes modelos de planificación estratégica desarrollados por el autor [13] y [14], se plantea en este trabajo dar continuidad a las hipótesis de estos modelos y aplicar al ámbito empresarial la metodología desarrollada por Sumpsi et al. [7], en la que, a partir de los resultados obtenidos, permite evaluar el cumplimiento de los objetivos y la estrategia seguida por el decisor. En general, dado que para una empresa cualquier decisión financiera implicará un balance o equilibrio entre elementos contrapuestos con diferentes criterios (por ejemplo, ampliar el dividendo respecto a mejorar la financiación propia; aumentar el apalancamiento financiero respecto de la solvencia o elegir entre financiación con recursos propios o ajenos), se plantea en este artículo una situación de notable interés práctico para las técnicas de decisión tras observar los resultados obtenidos por las empresas cuando se encuentran diferentes criterios en conflicto.

\section{Metodología: formulación matemática de un modelo de análisis de comportamiento estratégico}

La formulación general de un problema multicriterio en el que se plantean q objetivos relevantes en conflicto suele plantearse de la siguiente forma:

$$
\begin{gathered}
\operatorname{Eff}\left[f_{1}(\boldsymbol{x}), f_{2}(\boldsymbol{x}), \ldots, f_{q}(\boldsymbol{x})\right] \\
\text { Sujeto a } \boldsymbol{x} \in \boldsymbol{F}
\end{gathered}
$$


Donde:

- Eff es un operador que indica la búsqueda de soluciones eficientes o Pareto óptimas.

- $\boldsymbol{x}$ es el vector de variables de decisión del problema.

- $f j_{j}(\boldsymbol{x})$ la expresión matemática del objetivo j-ésimo.

- $\boldsymbol{F}$ es el conjunto factible, o aquellas soluciones que cumplen las restricciones del problema.

Para el caso particular de programación por metas ponderadas, se pueden definir además los siguientes inputs:

- $w_{j}$ : peso asociado a la prioridad del objetivo j-ésimo.

- $f_{j}^{*}$ valor ideal u óptimo del objetivo $j$-ésimo.

- $f_{j}$ : valor observado por el objetivo $j$-ésimo.

- $f_{i j}$ : valor obtenido por el objetivo $j$-ésimo, cuando el objetivo $i$-ésimo es optimizado $\left(f_{i j}=f_{j}\left(x^{\star}\right)\right)$.

- n $p_{i}$ : desviaciones positiva y negativa del $j$-ésimo objetivo respecto del valor observado.

El problema multiobjetivo de planificación estratégica planteado por Martin et al. [13] se centra en cinco objetivos estratégicos principales de sostenibilidad a largo plazo:

$-f_{1}(\boldsymbol{x})=x_{1}+x_{2}+x_{3}+x_{4}$ (expansión).

$-f_{2}(\boldsymbol{x})=\frac{P+A+x_{1}+x_{2}+x_{3}}{E+x_{4}}$ (solvencia).

$-f_{3}(\boldsymbol{x})=\frac{P+A+x_{1}+x_{2}+x_{3}}{C+x_{2}}$ (valor teórico de la acción).

$-f_{4}(\boldsymbol{x})=x_{6}$ (dividendo).

$-f_{5}(\boldsymbol{x})=R\left(F+x_{5}\right)-i\left(E+x_{4}\right)$ (eficiencia operativa).

que son calculados a partir de seis variables de decisión de carácter estratégico asociados a los incrementos patrimoniales de la sociedad en su balance a largo plazo:

$-\mathrm{x}_{1}=$ incremento de las reservas.

$-\mathrm{x}_{2}=$ incremento del capital social.

$-x_{3}=$ incremento de las amortizaciones.

$-\mathrm{x}_{4}=$ incremento del exigible a largo.

$-x_{5}=$ incremento del inmovilizado material.

$-\mathrm{x}_{6}=$ dividendo que se va a repartir.

y a partir de los siguientes parámetros que muestran la situación inicial del balance de la sociedad y su cuenta de resultados operativa esperada para el ejercicio:

- $P=$ patrimonio neto inicial de la sociedad.

- $A=$ amortización acumulada inicial. 
- $E=$ exigible a largo plazo inicial.

$-C=$ capital suscrito inicial.

- $I=$ inmovilizado bruto inicial, sin amortización.

- $G=$ resultado bruto del ejercicio, sin amortizaciones, intereses e impuestos.

- $R=$ rentabilidad sobre inmovilizado de la ganancia antes de impuestos.

- $R I N=$ tasa de retorno neta sobre inmovilizado después de impuestos.

- $T=$ impuesto sobre beneficios.

$-i=$ coste financiero.

$-\lambda=$ máximo coeficiente legal de depreciación del activo.

- $\mu$ = mínimo nivel de capital distribuido como dividendo.

El modelo plantea las siguientes restricciones para mantener la sostenibilidad a largo plazo de la empresa:

- La solvencia de la compañía debe ser superior al valor previo de solvencia: $f_{2}(\boldsymbol{x}) \geq$ $\frac{P+A}{E}$.

- El valor teórico de la acción debe ser creciente: $f_{2}(\boldsymbol{x}) \frac{P+A}{C}$.

- La compañía debe distribuir un dividendo mínimo, al menos superior a un $\mu$ por ciento del capital, dependiendo de cada sociedad: $x_{6} \geq \mu C$.

- El coste anual de amortización no debe superar los límites legales establecidos: $x_{3} \leq \lambda l$.

- El incremento anual de los activos debe ser cubierto con recursos permanentes, esto es, incrementos de capital, reservas y deuda: $x_{1}+x_{2}+x_{4}-x_{5}=0$.

- La ganancia bruta de la compañía después de impuestos debe ser distribuida en reservas y dividendo: $\left(G-x_{3}\right)-T\left(G-x_{3}\right)=x_{1}+x_{6}$.

- La tasa interna de retorno del beneficio sobre el activo después de impuestos debe ser superior a un cierto nivel: $\frac{(1-T)\left(G-x_{3}\right)}{\left(F+x_{5}\right)} \geq R I N$.

- Las variables de decisión no pueden ser negativas, $\boldsymbol{x} \geq \mathbf{0}$; esto es: $x_{i} \geq 0(i=1$..6).

A partir de la formulación de un modelo multicriterio, Sumpsi et al. [7] plantearon estimar el comportamiento observado del decisor «como si» estuviera sujeto al modelo matemático a partir de la estimación individual de las preferencias mostradas en la matriz de pagos.

Dado que la matriz de pagos $\left[f_{i j}\right]=\left[f_{j}\left(X^{\star}\right]\right.$ es por definición aquella cuyas columnas $(j=$ 1..q) corresponden a los vectores asociados a la optimización individual de cada uno de los objetivos que cumplen las restricciones del problema,

$$
[M P]=\left[\begin{array}{cccc}
f_{11}=f_{1}\left(x^{* 1}\right)=f_{1}^{*} & f_{12}=f_{2}\left(x^{* 1}\right) & \cdots & f_{1 q}=f_{q}\left(x^{* 1}\right) \\
f_{21}=f_{1}\left(x^{* 2}\right) & f_{22}=f_{2}\left(x^{* 2}\right)=f_{2}^{*} & \cdots & f_{2 q}=f_{q}\left(x^{* 2}\right) \\
\vdots & \vdots & \cdots & \vdots \\
f_{q 1}=f_{1}\left(x^{*}\right) & f_{q 2}=f_{2}\left(x^{*}\right) & \cdots & f_{q q}=f_{q}\left(x^{*}\right)=f_{q}^{*}
\end{array}\right]
$$


su trasposición permite plantear un sistema de ecuaciones tal que si el vector de preferencias w fuera canónico en su componente j-ésima (por ejemplo, si $\boldsymbol{w}=[1,0, . .0]^{\top}$ ), la solución obtenida por este sistema correspondería exactamente a la optimización del objetivo j-ésimo (por ejemplo, $f_{j}\left(x^{* 1}\right)$ ).

$$
\underbrace{\left[\begin{array}{cccc}
f_{11}=f_{1}{ }^{*} & f_{21} & \ldots & f_{q 1} \\
f_{12} & f_{22}=f_{2}^{*} & \ldots & f_{q 2} \\
\vdots & \vdots & \ldots & \vdots \\
f_{1 q} & f_{2 q} & \ldots & f_{q q}=f_{q}^{*}
\end{array}\right]}_{[M P]^{T}}\left[\begin{array}{c}
1 \\
0 \\
\vdots \\
0
\end{array}\right]=\underbrace{\left[\begin{array}{c}
f_{11}=f_{1}^{*} 1 \\
f_{12} \\
\vdots \\
f_{1 q}
\end{array}\right]}_{f\left(x^{* 1}\right)}
$$

Por tanto, una combinación lineal de estas posibles soluciones asociada a una diferente ponderación de las preferencias w generaría el siguiente sistema de ecuaciones:

$$
\underbrace{\left[\begin{array}{cccc}
f_{11}=f_{1}^{*} & f_{21} & \ldots & f_{q 1} \\
f_{12} & f_{22}=f_{2}^{*} & \ldots & f_{q 2} \\
\vdots & \vdots & \ldots & \vdots \\
f_{1 q} & f_{2 q} & \ldots & f_{q q}=f_{q}^{*}
\end{array}\right]}_{[M P]^{T}}\left[\begin{array}{c}
w_{1} \\
w_{2} \\
\vdots \\
w_{q}
\end{array}\right]=\underbrace{\left[\begin{array}{c}
f_{1} \\
f_{2} \\
\vdots \\
f_{q}
\end{array}\right]}_{f}
$$

que define implícitamente aquella combinación de pesos $\boldsymbol{w}=\left(w_{1}, \ldots, w_{q}\right)$ que, partiendo de las preferencias mostradas por el decisor en la matriz de pagos, genera el vector de observaciones $\boldsymbol{f}=\left(f_{1}, \ldots, f_{q}\right)$.

Este sistema, habitualmente, no tiene solución debido a las restricciones del problema y al conflicto que existe entre los objetivos. Sin embargo, cabe la posibilidad de buscar una solución aproximada bajo un entorno de programación por metas considerando los valores observados como metas que se van a alcanzar por ambos lados. En este caso, la función de logro correspondería a la minimización de la suma de las variables de desviación normalizadas al valor observado en cada uno de los objetivos.

Por tanto, la formulación del problema de análisis de comportamiento llevaría a resolver el siguiente sistema de ecuaciones:

$$
\operatorname{Min}\left[\frac{n_{1}+p_{1}}{f_{1}}+\frac{n_{2}+p_{2}}{f_{2}}+\ldots+\frac{n_{q}+p_{q}}{f_{q}}\right]
$$

Sujeto al sistema de ecuaciones:

$$
\left.\begin{array}{c}
\underbrace{\left[\begin{array}{cccc}
f_{11}=f_{1}^{*} & f_{21} & \ldots & f_{q 1} \\
f_{12} & f_{22}=f_{2}^{*} & \ldots & f_{q 2} \\
\vdots & \vdots & \ldots & \vdots \\
f_{1 q} & f_{2 q} & \ldots & f_{q q}=f_{q}^{*}
\end{array}\right]}_{[M P]^{T}} \\
w_{1}+w_{2}+\ldots+w_{q}=1 \\
w_{q}
\end{array}\right]+\left[\begin{array}{c}
w_{1} \\
w_{2} \\
\vdots \\
n_{q}-p_{q}
\end{array}\right]+\underbrace{\left[\begin{array}{c}
n_{1}-p_{1} \\
n_{2}-p_{2} \\
\vdots \\
f_{q}
\end{array}\right]}_{f}
$$


En general, la última restricción $\mathrm{w}_{1}+\mathrm{w}_{2}+\ldots+\mathrm{w}_{\mathrm{q}}=1$ se plantea para que el conjunto de preferencias esté normalizado. La resolución de este sistema permitiría, a partir de resultados observados y de la solución aproximada, identificar el comportamiento y la elección de preferencias del decisor.

\subsection{Ejemplo de aplicación}

Para mostrar la aplicabilidad de este modelo analítico de predicción (4) en el ámbito de la economía digital, se ha seleccionado como caso de aplicación a la firma Apple Inc., líder de mercado y firma de mayor capitalización a lo largo de los últimos años. Para este análisis, se han escogido los últimos cuatro ejercicios con los siguientes datos seleccionados:

Tabla 1. Valores de los parámetros exógenos del modelo predictivo para Apple Inc.

\begin{tabular}{|c|c|c|c|c|c|c|}
\hline & Apple Inc. & 2018 & 2017 & 2016 & 2015 & 2014 \\
\hline $\mathrm{I}_{1}$ & Activos de largo plazo amortizables & 82.683 & 70.257 & 57.482 & 46.242 & 34.700 \\
\hline $\mathrm{I}_{2}$ & Otros activos no amortizables & 210.593 & 184.601 & 174.603 & 138.542 & 112.938 \\
\hline 1 & Total inmovilizado bruto & 293.276 & 254.858 & 232.085 & 184.784 & 147.638 \\
\hline \multirow[t]{2}{*}{ A } & Amortización acumulada & 46.602 & 40.041 & 31.118 & 21.476 & 13.924 \\
\hline & Total activo no corriente neto & 246.674 & 214.817 & 200.967 & 163.308 & 133.714 \\
\hline $\mathrm{C}$ & Capital aportado & 35.867 & 31.251 & 27.416 & 23.313 & 19.764 \\
\hline $\mathrm{P}$ & Patrimonio neto (capital y reservas) & 134.047 & 128.249 & 119.355 & 111.547 & 123.549 \\
\hline \multirow[t]{2}{*}{$E$} & Exigible a largo plazo & 140.458 & 114.431 & 90.380 & 56.844 & 39.793 \\
\hline & $\begin{array}{l}\text { Total exigible a largo y patrimonio } \\
\text { neto }\end{array}$ & 274.505 & 242.680 & 209.735 & 168.391 & 163.342 \\
\hline G & EBITDA & 70.744 & 69.824 & 81.730 & 60.503 & 55.759 \\
\hline $\mathrm{R}$ & Rentabilidad bruta & $24 \%$ & $26 \%$ & $39 \%$ & $36 \%$ & $39 \%$ \\
\hline $\mathrm{T}$ & Tipo impositivo & $25 \%$ & $26 \%$ & $26 \%$ & $26 \%$ & $26 \%$ \\
\hline$K_{f}$ & Coste financiero & $2,5 \%$ & $2,8 \%$ & $3,8 \%$ & $3,2 \%$ & $6,1 \%$ \\
\hline $\mathrm{U}$ & Tasa dividendo sobre capital & $35 \%$ & $38 \%$ & $42 \%$ & $47 \%$ & $53 \%$ \\
\hline$W_{1}$ & $\begin{array}{l}\text { Tasa amortización sobre inm. } \\
\text { amortizable }\end{array}$ & $13 \%$ & $17 \%$ & $23 \%$ & $23 \%$ & $25 \%$ \\
\hline
\end{tabular}

Fuente: Thompson Reuters Eikon [16]. 


\section{Resultados}

El primer paso es obtener las matrices de pagos (1) asociadas a cada uno de los ejercicios:

Tabla 2. Matrices de pagos obtenidos para Apple Inc.

\begin{tabular}{|c|c|c|c|c|c|c|c|c|c|c|c|}
\hline \multicolumn{6}{|c|}{2018} & \multicolumn{6}{|c|}{2017} \\
\hline & 21 & z2 & 23 & 24 & 25 & & 21 & $z 2$ & z3 & 24 & 25 \\
\hline$z 1$ & 39.908 & 1,819 & 6,662 & 13.150 & 65.525 & $z 1$ & 43.536 & 2,147 & 7,076 & 8.832 & 68.950 \\
\hline z2 & 39.908 & 1,819 & 6,662 & 13.151 & 65.525 & z2 & 43.536 & 2,147 & 7,076 & 8.832 & 68.950 \\
\hline z3 & 39.908 & 1,819 & 6,662 & 13.151 & 65.525 & 23 & 43.536 & 2,147 & 7,076 & 8.832 & 68.950 \\
\hline z4 & - & 1,471 & 5,385 & 53.058 & 56.266 & z4 & - & 1,665 & 5,488 & 52.368 & 57.631 \\
\hline 25 & 39.908 & 1,819 & 6,662 & 13.151 & 65.525 & 25 & 43.536 & 2,147 & 7,076 & 8.832 & 68.950 \\
\hline Ideal & 39.908 & 1,819 & 6,662 & 65.525 & 65.525 & Ideal & 43.536 & 2,147 & 7,076 & 52.368 & 68.950 \\
\hline Anti-ideal & - & 1,471 & 5,385 & 56.266 & 56.266 & Anti-ideal & - & 1,665 & 5,488 & 8.832 & 57.631 \\
\hline Real & 31.825 & 1,286 & 5,037 & 12.563 & 61.211 & Real & 32.945 & 1,471 & 5,385 & 11.965 & 58.829 \\
\hline \multicolumn{6}{|c|}{2018} & \multicolumn{6}{|c|}{2017} \\
\hline & 21 & 22 & 23 & 24 & 25 & & 21 & $\mathrm{z2}$ & 23 & 24 & 25 \\
\hline$z 1$ & 30.265 & 2,873 & 7,004 & 29.948 & 79.445 & $z 1$ & 24.562 & 4,071 & 8,199 & 20.210 & 58.877 \\
\hline z2 & 30.265 & 2,873 & 7,004 & 29.949 & 79.445 & z2 & 24.562 & 4,071 & 8,199 & 20.210 & 58.877 \\
\hline$z 3$ & 30.265 & 2,873 & 7,004 & 29.949 & 79.445 & $z 3$ & 24.562 & 4,071 & 8,199 & 20.210 & 58.877 \\
\hline$z 4$ & - & 2,340 & 5,706 & 60.214 & 67.944 & z4 & - & 3,455 & 6,956 & 44.772 & 50.281 \\
\hline 25 & 30.265 & 2,873 & 7,004 & 29.948 & 79.445 & 25 & 24.562 & 4,071 & 8,199 & 20.210 & 58.877 \\
\hline Ideal & 30.265 & 2,873 & 7,004 & 60.214 & 79.445 & Ideal & 24.562 & 4,071 & 8,199 & 44.772 & 58.877 \\
\hline Anti-ideal & - & 2,340 & 5,706 & 29.948 & 67.944 & Anti-ideal & - & 3,455 & 6,956 & 20.210 & 50.281 \\
\hline Real & 41.344 & 1,665 & 5,489 & 11.431 & 70.327 & Real & 5.049 & 2,340 & 5,706 & 11.031 & 52.192 \\
\hline
\end{tabular}

La aplicación del modelo de aproximación (4) sobre las matrices de la tabla 2 genera los siguientes vectores de preferencias:

Tabla 3. Resultados del modelo de comportamiento para Apple Inc.

\begin{tabular}{|c|c|c|c|c|c|c|}
\hline Resultados & 2018 & 2017 & 2016 & 2015 & Promedio & $\begin{array}{c}\text { Promedio } \\
\text { exc 2015 }\end{array}$ \\
\hline w1 & - & - & - & - & - & - \\
\hline w2 & - & - & - & - & - & - \\
\hline w3 & - & - & - & - & - & - \\
\hline w4 & - & 0,071 & - & 0,794 & 0,216 & 0,024 \\
\hline w5 & 1,000 & 0,929 & 1,000 & 0,205 & 0,784 & 0,976 \\
\hline $\begin{array}{c}\text { Error cuadrático } \\
\text { medio }\end{array}$ & $11,8 \%$ & $11,9 \%$ & $36,4 \%$ & $53,3 \%$ & $28,3 \%$ & $20,0 \%$ \\
\hline
\end{tabular}


El análisis de los resultados obtenidos (tabla 3) muestra unas cotas razonables de error siempre que los valores reales estén contenidos dentro del rango ideal-antiideal. Cuando los datos no cumplen esta condición, por ejemplo, en el año 2015, el error de predicción se eleva notablemente. La razón principal de esta situación se debe a que el modelo plantea como hipótesis de partida la sostenibilidad a largo plazo, concretada en la restricción de la mejora anual de los ratios de solvencia y creación de valor. Cuando la hipótesis no se cumple, el valor obtenido se encuentra fuera del rango de aproximación y no es ajustable dentro del rango de compromiso de la matriz de pagos, generando un error de aproximación en las variables de desviación.

En segundo lugar, cabe indicar la gran correlación mostrada por la matriz de pagos en el conjunto de los objetivos, excepto en dividendo. La maximización del objetivo dividendo genera los valores antiideales de la matriz, mientras que los valores ideales se obtienen de manera simultánea optimizando cualquiera de los restantes objetivos. Este resultado podría ser esperable a priori, ya que, si los recursos generados se emplean en retribuir directamente al accionista, se reduce la autofinanciación y el potencial de crecimiento de la empresa que se concreta en el resto de objetivos.

En tercer lugar, puede observarse en los resultados un comportamiento similar a lo largo de los años priorizando la eficiencia y rentabilidad frente al objetivo dividendo. La ponderación que mantiene el objetivo de eficiencia llega al $97 \%$ en el promedio de los tres últimos años.

Por último, es importante reseñar la mejora de estos resultados con respecto al supuesto equipreferencial $w_{i}=0,2(i=1 . .5)$, habitual en caso de carecer de información acerca de las preferencias del decisor. El error de predicción en este caso aumenta en comparación con los resultados obtenidos con la metodología de aproximación por metas.

\section{Conclusiones}

A lo largo del presente trabajo, se ha intentado aplicar una metodología de análisis de comportamiento observado a un entorno de planificación financiera estratégica. Como primera conclusión, puede indicarse que resulta posible aplicar estas técnicas que permiten verificar que, una vez observados los resultados ofrecidos por las empresas, se puede cuantificar su estrategia a partir de los resultados observados.

1. La consideración de estos resultados induce a pensar que, para analizar adecuadamente una compañía y poder realizar correctas predicciones de futuro acerca de la misma, resulta imprescindible comprender el comportamiento previo del equipo decisor, para, posteriormente, realizar las propuestas estratégicas que ponderen de la mejor forma posible sus preferencias. En consecuencia, este modelo de análisis de comportamiento permite identificar estas preferencias y plantear los objetivos futuros de una forma coherente planteando escenarios en los que las empresas puedan asumir criterios alternativos a los de maximización del beneficio como únicos objetivos directores de su estrategia.

2. La calidad de esta predicción dependerá en gran medida del modelo matemático aplicado sobre el que se define la matriz de pagos y el esquema de análisis de comportamiento. Si las directrices de la empresa no se adaptan al modelo por ser excesivamente rígido 
en sus planteamientos, o bien porque mantiene un comportamiento errático en sus resultados, este modelo generará resultados con altas cotas de error.

3. Como futuras líneas de esta investigación, cabría pensar en la extensión de este modelo de análisis de comportamiento a otros modelos de planificación financiera más precisos, que se adapten mejor al comportamiento seguido por la empresa, ya sean a largo plazo o en el balance completo de la empresa. Por ejemplo, el modelo plantea como hipótesis una mejora de la solvencia, cuando en el caso de aplicación la empresa seleccionada aumenta cada año su apalancamiento financiero.

\section{Bibliografía}

1. Koopmans, T. C. (1951). «An analysis of Production as an Efficient Combination of Activities». John Wiley and Sons.

2. Kuhn, H. W. y Tucker, A. W. (1951). «Nonlinear Programming in J. Neyman (ed.)». Proceedings of the Second Berkeley simposium on Mathematical Statistical and Probability. University of California Press-Berkeley.

3. Charnes, A. y Cooper, W. W. (1961). Management Models and Industrial Applications of Linear Programming. John Wiley and Sons.

4. Yu, P. L. (1973). «Introduction to Domination Structures in Multicriteria Decision Problems», en: Cochrane, J. L. y Zeleny, M. (editores). Multiple Criteria Decision Making, University of South Carolina Press: $249-261$.

5. Zeleny M. (1973). «Compromise Programming», en: Cochrane, J. L. y Zeleny M. (editores). Compromise Programming in Multiple Criteria Decision Making. University of South Carolina Press: 262-301.

6. André, F. J. y Romero, C. (2008). «Computing compromise solutions: On the connections between compromise programming and composite programming». Applied Mathematics and Computation, vol. 195: 1-10.

7. Sumpsi, J. M., Amador, F. y Romero, C. (1996). «On farmers' objectives: A multi-Criteria approach». European Journal of Operational Research, vol. 96: 64-71.

8. Kvanli, A. H. (1980). «Financial Planning Using Goal Programming». Omega, The International Journal of Management Sciences, vol. 8: 207-218.

9. Batson, R. G. (1989). «Financial Planning Using Goal Programming». Long Range Planning, vol. 22: 112-120.

10. Goedhart, M. H. y Spronk, J. (1995). «Financial planning with fractional goals». European Journal of Operational Research, vol. 82: 111-124.

11. Maranas, D., Androulakis, I. P., Floudas, C. A., Berger A. J. y Mulvey J. M. (1997). «Solving long-term financial planning problems via global optimization». Journal of Economic Dynamics and Control, vol. 21: 1405-1425.

12. Tarrazo, M. y Gutiérrez, L. (2000). «Economic expectations, fuzzy sets and financial planning». European Journal of Operational Research, vol. 126: 89-105.

13. Martín, M. A., Cuadrado, M. L. y Romero, C. (2011). "Computing efficient financial strategies: An extended compromise programming approach». Applied Mathematics and Computation, doi:10.1016/j. amc.2011.02.10.

14. Martín, M. A., Romero, M. S. y Romero, L. (2017). «Un modelo de Planificación Financiera Sostenible, a través de un proceso de optimización». Revista Internacional Jurídica y Empresarial, vol. 1: 59-73.

15. Zopounidis, C., Galariotis, E., Doumpos, M., Sarri, S. y Andriosopoulos, K. (2015). «Multiple criteria decision aiding for finance: An updated bibliographic survey». European Journal of Operational Research, vol. 247: 339-348.

16. Base de datos Thompson Reuters Eikon (2018). 